\title{
Plasma Extraction of Metals in Space
}

\author{
Peter J Schubert* \\ Indiana University-Purdue University Indianapolis, Indianapolis, Indiana, USA
}

Submission: November 18, 2019; Published: November 27, 2019

*Corresponding author: Peter J Schubert, Indiana University-Purdue University Indianapolis, Indianapolis, Indiana, USA

\begin{abstract}
Extraction of purified metals from extraterrestrial materials can be accomplished in several ways, such as beneficiation, hydrogen reduction, recovery of spent rockets, direct melting of iron meteorites, and plasma isotope separation. Presented here is a method for multiple simultaneous extraction of multiple metals and metalloids from regolith. Two patented approaches are described which can operate on a planetary surface, or in the microgravity environment of orbit. This approach to isotope separation applies to regolith fines but is advantageously applied to the effluent of a patented oxygen extraction method. In this way, a plurality of valuable raw materials can be obtained with a single system, suitable for operation on the Moon or at the surface of an asteroid. Silicon is of interest for studies of purity due to its importance in photovoltaics. A silicon-aluminum aerospace alloy can be produced directly, called Silumin, which has value in construction of habitats and space craft in space. Silicon can also be combined with carbon to form the wide-bandgap semiconductor SiC from which high-power and radiation-tolerant power transistors can be fabricated. Furthermore, this method lends itself to additive manufacturing whereby specific shapes of purified metals can be formed directly from the plasma extraction process.
\end{abstract}

Keywords: Extraterrestrial materials; Space solar power; Gravity; Radiation; Vacuum; Asteroids; Ammonia

\section{Introduction}

In situ resource utilization (ISRU) involves extraction of raw materials and the making of value-added components from materials found in space. Earth launch requires enormous energies and will always be costly. Employing indigenous extraterrestrial materials enables greater exploitation of space resources, which can be used in an orbital economy, or delivered back to markets on the home world. One lucrative prospect is to build vast solar farms which beam power gigawatts of power wirelessly to customers on the ground. Known as space solar power (SSP) $[1,2]$ the economics at large scale is highly favorable if the photovoltaics, structural elements, wires, and power electronics can be fabricated from in situ resources [3]. This study explores a new method which is the subject of three US patents by the author [4-6].

Space resources include sunlight, vacuum, gases, variable gravity, radiation, and solid matter in the form of asteroids and planetary bodies. Asteroids are grouped into categories and may include: low atomic weight volatiles such as water, methane, and ammonia; carbon-rich amalgams with silicate-based rocks; stony iron bodies with substantial amounts of relatively pure transition metals; and rocky asteroids with mostly silicatebased minerals containing other elements including, but not limited to: calcium, magnesium, aluminum, and titanium [7-10]. Terrestrial methods of mining and extraction generally rely on strong gravity and water. Without these, mining and extraction in space is difficult. Delivery of water into orbit is expensive, and production of high gravity (e.g. $9.8 \mathrm{~m} / \mathrm{s} 2$ ) requires a large centrifuge or rocket accelerations, so that extractive techniques call for a thorough re-thinking for ISRU.

Several extractive methods have been developed for use in space, such as oxygen for life support, water for rocket fuel, titanium for structures, ceramics and refractories for building blocks and roadways, and iron for railroads. This paper studies an energetic means by which multiple, simultaneous streams of pure metals can be extracted from the broken and shattered surface rock dust known as regolith. Regolith covers all airless bodies in space. Within this powdery tuff can be found a wide variety of chondrites, spherules, brecciated minerals, and free iron. The goal of this plasma extraction method is to heat such regolith enough to disaggregate into individual atoms which can be separated by mass. The energy penalty is high; however, the payoff is in a high yield of several useful raw materials in a manner well-suitable to further value-added operations.

\section{Apparatus}

High temperature is an equalizer for regolith because at sufficiently high temperatures, physical morphology is irrelevant. However, molten regolith is an excellent solvent, and will compromise most refractory crucibles. Liquid regolith simulant in yttria-stabilized zircon will melt together into a 
puddle. The only refractory known to withstand molten silicates is thorium dioxide [11]. Thoria has the highest melting point of any metal oxide and has no transition temperatures up to 2950 $\mathrm{K}[12,13]$. When heated to these temperatures, molten regolith will spontaneously devolve oxygen gas. Oxygen can be captured by absorbents using a deLaval nozzle, drift tube, and expansion bell [14], as seen in the center of Figure 1. The effluent of oxygen extraction is ballistic nanometer-sized reduced-oxygen minerals (e.g. $\mathrm{SiO}, \mathrm{FeO}$ ), and this stream is the input to the isotope separation apparatus $[15,16]$.

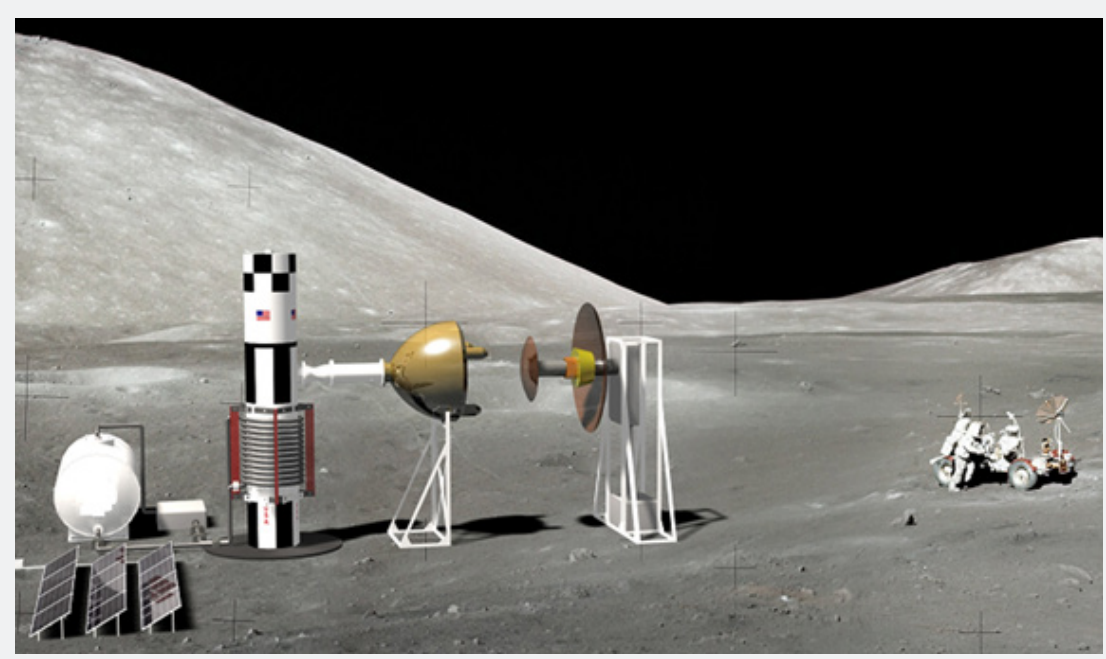

Figure 1: Lunar Facility to Extract Oxygen, Silicon, and Metals from Regolith.
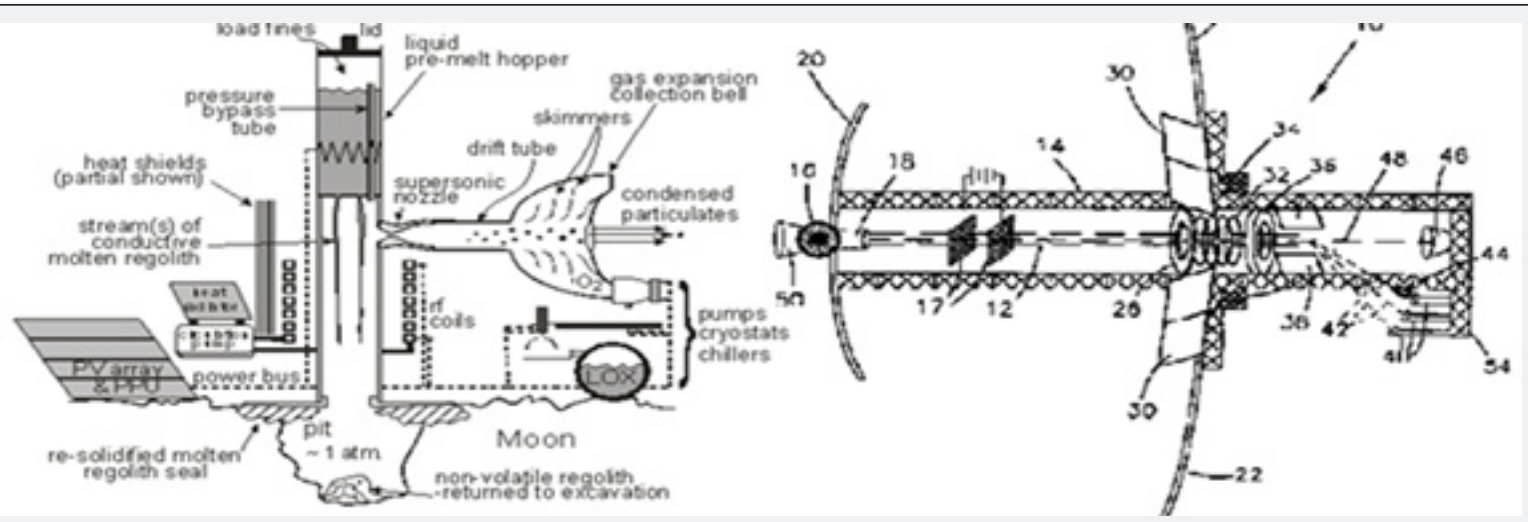

Figure 2: Cross-Section Schematic of Oxygen Dust Roaster and Isotope Separator in Series.

Figure 2 shows a cross-section schematic of the combined oxygen extraction "dust roaster" and the plasma metals extractor "isotope separator" in the same configuration as Figure 1. Material enters at the top left and exits at the gas expansion bell, and then at the metals collection receptacles (items 41). Operation of the oxygen dust roaster is to first liquefy the regolith via heater elements exterior to the thoria containment vessel. Apertures in the bottom of the liquefaction vessel admit magma to a vertical (thoria) tube which is heated by radiofrequency (rf) energy to engage the conductive liquid [17] as it falls in lunar gravity and raises the temperature to that of a vapor. Note that in zero gravity this portion would require a centrifugally rotating apparatus to provide the appropriate artificial gravity (approximately 0.18 of earth's). Not all mineral species will be vaporized, so that oxides of calcium and magnesium will fall into a sealed pit from which it can be later extracted to make refractory bricks. The supersonic deLaval nozzle in the vertical tube provides escape for vaporized materials, which pass through a short drift tube to cool slightly. This cooling region is on the order of several centimeters, during which the sub-oxide minerals will begin to coalesce, like the exhaust plume of a solid-fuel rocket motor. Oxygen liberated from the minerals will experience expansion upon reaching the gas collection bell and is directed to absorption collectors made from praesidium-cerium oxide. These collectors must be periodically detached to thermally release their load of pure oxygen [18]. The coalescing minerals become ballistic, meaning they are no longer entrained by the gases, and escape the gas collection bell through an aperture. The velocity of this stream carries material into the isotope separator. Note that the isotope separator is designed to also accept regolith fines which have been beneficiated to sub-micron dimension but without the preliminary step of extracting the oxygen. In that case, an optional impeller is provided to deliver the initial kinetic velocity to these particles (item 10 in Figure 2). 
The isotope separator works in a similar manner to a mass spectrometer, wherein ionized elements are accelerated in a plasma beam which is passed through a transverse electric or magnetic field (item 38 in Figure 2) to deflect the ions. Lighter ions are deflected further than heavier ions, so the result is a spectrum of elements (dotted lines 42 in Figure 2), with those having higher atomic numbers clustered closer to the beam axis, and the low atomic number elements deflected at larger and larger angles. Because of this, the separation efficiency is greatest for metals such as silicon and aluminum. Hot ions impinge on the collection receptacles, which are optionally cooled to accrete amorphous masses of purified metals. The metals must be separated from the receptacle, which may be advantageously formed from the refractory $\mathrm{CaO}$ and $\mathrm{MgO}$ mentioned above. Nonionized material will not be deflected and can be captured in receptacle 46 . The workpiece receiving non-ionized material can be manipulated in orientation to produce convex shapes of this slag. Such items may find use as building or shielding materials for space settlements.

\section{Methods}

To study plasma dissociation of sub-oxide minerals, the silicon monoxide ( $\mathrm{SiO}$ ) molecule is most prevalent from regolith, and important because of the silicon it contains. The dissociation energy of SiO is $460 \mathrm{~kJ}$ per mole of the molecule. This value can be used to estimate the minimum power to break apart molecules into their constituent atoms as a function of beam flow rate in moles/second. These atoms must then be ionized, and the ionization energy for silicon is $786 \mathrm{~kJ} / \mathrm{mol}$. The moving beam of ionized elements represents a current, which, induced onto the stream to motivate the ions in the direction intended, adds a third component of electrical power for the most energyintensive operations in the isotope separator. For a reference case of $11.5 \mathrm{~g} / \mathrm{sec}$ of regolith (total), this requires a primary power of $139 \mathrm{~kW}$, with silicon extraction of $10.4 \mathrm{~kg} /$ hour.

Separation of isotopes goes by the quotient of electric charge by ion mass, called the charge-mass ratio $(q / m)$. The Lorentz force deflects ions from a collimated beam, creating a geometrically simple separation, either by radius in the case of a transverse magnetic field, or by angle in the case of a transverse electric field. Figure 3 shows the elements adjacent to silicon, along with their global average abundance on the moon. The ionization energy mentioned above must be imparted to each ion for separation to occur, so the first ionization energy is also listed in Figure 3. Figure 3 does not tell the entire story of isotope separation. There can exist resonances wherein the second ionization $(\mathrm{q}=+2)$ of an element twice as massive can coincide with the stream of a singly ionized lighter element. For example, heavy iron (Fe58) is 1.999 the mass of Si29; fortunately, the second ionization energy for iron is $1561.9 \mathrm{~kJ} / \mathrm{mol}$. In practice, the amount of energy imparted by rf fields is to be carefully calibrated to avoid resonances.

\begin{tabular}{|l|r|r|r|r|}
\hline \multicolumn{1}{l}{ ISOTOPE } & \multicolumn{1}{c|}{ AMU } & \multicolumn{1}{c|}{ ABUNDANCE PERCENT Ionization Energy } \\
\hline $\mathrm{P}$ & 30.9737 & 100 & 0.1 & $1011.7 \mathrm{~kJ} / \mathrm{mol}$ \\
\hline $\mathrm{Si}-28$ & 27.97693 & 92.2297 & 16.6 & $786.4 \mathrm{~kJ} / \mathrm{mol}$ \\
\hline $\mathrm{Si}-29$ & 28.97649 & 4.6832 & 16.3 & $786.4 \mathrm{~kJ} / \mathrm{mol}$ \\
\hline $\mathrm{Si}-30$ & 29.97377 & 3.0875 & 16.3 & $786.4 \mathrm{~kJ} / \mathrm{mol}$ \\
\hline $\mathrm{Al}$ & 26.98 & 100 & 10.1 & $577.6 \mathrm{~kJ} / \mathrm{mol}$ \\
\hline
\end{tabular}

Figure 3: Atomic Mass Units (AMU) for Silicon and Neighboring Elements, including their Relative Abundance in Lunar Regolith (Averaged Between Highland and Mare), With 1st lonization Energy.

Results

Three means of obtaining pure elements are:

a) physical apertures, with the drawback of buildup

b) isolated receptacles

c) capturing an entire spectrum of element and excising those slivers of desired purity

Silicon is bracketed by phosphorus and aluminum. Both $\mathrm{P}$ and $\mathrm{Al}$ are dopants in semiconductor silicon, $\mathrm{p}$-type and n-type respectively, therefore silicon intended for photovoltaics or integrated circuits should be free of these elements. Assuming method (2) above, the results of $\mathrm{q} / \mathrm{m}$ from a transverse electric field are shown in Figure 4. The left plot shows the separation by $\mathrm{q} / \mathrm{m}$ "bins" for $\mathrm{P}, \mathrm{Si}$, and $\mathrm{Al}$ with per mol ionization energy less than $1100 \mathrm{~kJ}$. The right plot in Figure 4 shows what happens if ionization energy exceeds that of $\mathrm{Fe}$ and $\mathrm{Ni}$ but remains lesser than the (generally much greater) third ionization energy of elements three times as massive as $\mathrm{Si}$. It is seen that the purity of each element depends on the overlap of similar isotope $\mathrm{q} / \mathrm{m}$ ratios, as well as the fineness of the aperture dividing one isotope from the next. Another factor is the spread in the velocity distribution. Although this method of plasma separation of isotopes is not in equilibrium, tendencies in that direction will approach a Maxwell-Boltzmann distribution of velocities, which has an appreciable dispersion. This issue can be partly ameliorated by beam profile techniques, or by velocity filter mechanisms. Beam diameter is another parameter which will affect purity because of parallax between ions emerging from different points across a beam cross section. It can be appreciated that a trade exists between yield and purity. Furthermore, selfshielding by the beam of material will raise the energy required for full dissociation and ionization. Thus, it can be further appreciated that there exists a trade space between throughput and efficiency. 


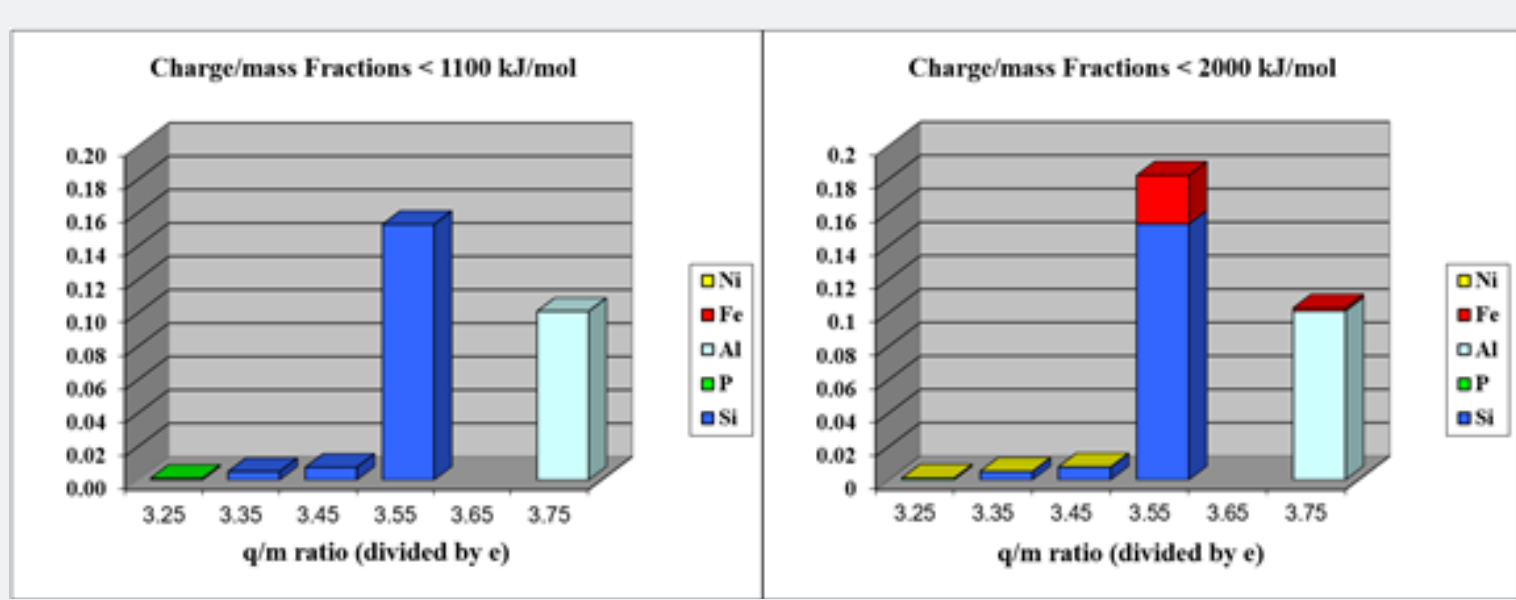

Figure 4: Isotope Separation Bins for first lonization (Left) and Second lonization (Right).

\section{Conclusion}

For beam ionizations below $1100 \mathrm{~kJ} / \mathrm{mol}$ a clean separation of silicon from other elements is possible. Pure aluminum is similarly accessible, as is the small amount of phosphorus found in most regolith samples. Together, these three elements can become the basis for complete solar cells and rudimentary integrated circuits. A mining operation producing these materials may find customers willing to produce solar power and computer chips in space. One example is the solar power satellite known as the "tin can" [19], with the potential to provide gigawatts of power to paying terrestrial customers. By combining the receptacles for $\mathrm{q} / \mathrm{m}$ ratios 3.55 and 3.75 , in various degrees of overlap, an aluminum alloy containing silicon ("Silumin") can be produced. Silumin has been used for aerospace applications and may be of interest to customers building habitats or spacecraft on the moon or at orbital platforms. Heavier elements such as iron, nickel and cobalt will not separate well, but may be blended in a fashion like the alloying of Silumin to make various steels and ferromagnetic metals of use in rail launchers and circumpolar railroads for remaining in constant sunlight while remaining on the moon. Further optimization work is planned through simulation and modeling tools to explore the trade spaces identified by this work.

\section{Statement}

The author declares no financial conflict of interest related to this work. Portions of this work were presented at the International Space Development Conference in Chicago, Illinois, US in 2010.

\section{References}

1. Schubert PJ (2005) A Novel Method for Element Beneficiation Applied to Solar Panel Production. Space Exploration, Albuquerque, NM.

2. Schubert PJ ((2006) Synergistic Construction Mechanisms for Habitats in Space Environs. International Space Development Conference las Angeles CA.

3. Schubert PJ (2009) Energy and Mass Balance for a Cislunar Architecture supporting SSP. AIAA SPACE 09 Pasadena CA.
4. Schubert PJ (2004) Process and apparatus for continuous-feed allisotope separation in microgravity using solar power. US.

5. Schubert PJ (2005) Process and apparatus for isotope separation in a low-gravity environment. US.

6. Schubert PJ (2008) Isotope separation process and apparatus therefor. US.

7. Lewis JS, Matthews MS, Guerrieri ML (1993) Resources of Near-Earth Space. (eds.), U. Arizona press.

8. Burbine TH (2017) Asteroids: Astronomical and Geological Bodies. Cambridge Planetary Science.

9. Williams RJ, Jadwick JJ (1980) Handbook of Lunar Materials. (eds.), NASA Ref. Pub.1057.

10. Turkevich AL (1973) The average chemical composition of the lunar surface. Proc. $4^{\text {th }}$ Lunar Sci Conf Suppl 4 Geochim Et Cosmochim Acta, pp. $1159-1168$.

11. Schubert PJ (2010) Materials Selection and Processing for Lunar Based Space Solar Power. Matls Challenges in Alt. and Renew. Energy, Cocoa Beach, FL.

12. Schubert PJ, Cunzeman K (2008) Ultra-High Temperature Materials for Lunar Processing. Am Soc of Engineering Educators Annual Conference Pittsburgh, PA.

13. Cunzeman K, Schubert PJ (2009) Survey of Ultra-High Temperature Materials for Applications Above 2000 K. AIAA SPACE 09, Pasadena, CA.

14. Burton RL, Schubert PJ, Rysanek F, Fenoglio P, Hutches M (2011) Oxygen Extraction Apparatus and Process, US.

15. Schick HL (1960) A Thermodynamic Analysis of the High-Temperature Vaporization Properties of Silica. Chem Rev 60: 331-362.

16. Shornikov SI, Archakav Yu I, Shults MM (1998) Mass Spectrometric Study of Vaporization and Thermodynamic Properties of Silicon Dioxide. I. Composition of the Gas Phase and Partial Vapor Pressures of the Molecular Forms over Silicon Dioxide. Russian J of General Chemistry 68(8): 1171-1177.

17. Ducret AC, Khetpal D, Sadoway DR (2002) Electrical Conductivity and Transference Number Measurements of $\mathrm{FeO}-\mathrm{CaO}-\mathrm{MgO}-\mathrm{SiO} 2$ Melts. Molten Salts, Proc Thirteenth Intl Symp ECS Pennington, NJ, pp. 347353. 
18. Schubert PJ (2007) A Novel Means for ISRU Oxygen Production. Space Resources Roundtable IX, Golden, CO.

This work is licensed under Creative Commons Attribution 4.0 License DOI: $10.19080 /$ IMST.2019.01.555568
19. Schubert PJ, Mukish KM, Shen MK, Pravinjit S (2016) Assembly and Operation of a "Tin Can" SPS. Int 1. Astro. Conf 2016, Guadalajara, Jalisco, Mexico.

Your next submission with Juniper Publishers will reach you the below assets

- Quality Editorial service

- Swift Peer Review

- Reprints availability

- E-prints Service

- Manuscript Podcast for convenient understanding

- Global attainment for your research

- Manuscript accessibility in different formats

( Pdf, E-pub, Full Text, Audio)

- Unceasing customer service

Track the below URL for one-step submission https://juniperpublishers.com/online-submission.php 